\title{
Charge Injection in Gold Ground Electrode Corona Charged Polyethylene Film: Surface Potential Decay and Corona Charging Current Measurement
}

\author{
Y. Zhuang, G. Chen and M. Rotaru \\ School of Electronics and Computer Science, University of Southampton \\ Southampton, SO17 1BJ, United Kingdom \\ yz205@ecs.soton.ac.uk
}

\begin{abstract}
Surface potential decay measurement is a simple and low cost tool to examine electrical properties of insulation materials. Bipolar charge injection has been verified by the measurement of space charge in the corona-charged sample using the pulsed electroacoustic method (PEA). In this paper, the surface potential decay of gold grounded electrode LDPE sample is compared with the aluminum grounded sample. The decay process observed by PEA and the corona charging result is presented in this paper. It can be clearly seen that the effect of using gold ground electrode is significant, it results in a smaller steady state corona charging current and a slower surface potential decay.
\end{abstract}

\section{INTRODUCTION}

Over the years, many interests have been shown in the surface potential decay of corona charged polymeric materials. It has become a well-known simple and low cost tool to examine electrical properties of dielectrics. The study of the potential decay in dielectric materials has a long history and is closely related to the wide application of corona charge dielectrics. The special cross-over phenomenon discovered in 1967 [7] showed that the initial potential of the sample decays more rapidly at higher field compared with lower field. Since then, the surface potential decay mechanism had become a very hot topic; however, the physical mechanism is not well understood. It also has been found that bipolar charge injection take place in corona charged LDPE films [2]. Charge injection from the bottom electrode was measured by using pulsed electro-acoustic method (PEA) and was proved by measuring the decay process for multiple layers sample.

\section{EXPERIMENTAL DETAILS}

In this paper, the surface potential decay of $55 \mathrm{~mm}$ diameter LDPE film with $50 \mu \mathrm{m}$ thickness was measured by JCI 140 static monitor with either $20 \mathrm{~nm}$ gold ground electrode (was metallized by K500X Sputter coater) or the normal aluminum ground. The corona charging current was observed by a volt-meter across a 10 $\mathrm{M} \Omega$ resistor. A needle-grid electrode corona charging system was used. A schematic diagram of the experiment set up is shown in figure 1 . The experiments were done with 2 min charging time with various charging voltages in a controlled environment where temperature and relative humidity were kept at $23{ }^{\circ} \mathrm{C}$ and $20 \%$, respectively. The influence of charge injection from the ground electrode on the surface potential decay and corona charging current has been studied.

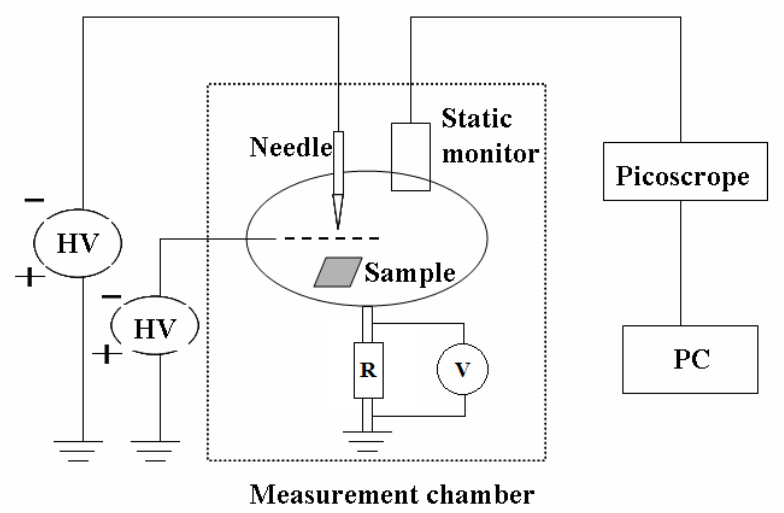

Figure 1. Experiment set up for corona charging and potential decay measurement system

\section{RESULTS AND DISCUSSION}

\section{A. Surface Potential Decay}

The surface potential measured by the static monitor is shown in figure 2 , it can be clearly seen that the gold ground affect the decay process for both higher field and lower field. The decay rate for $-2 \mathrm{kV}$ is reduced from $31 \%$ to $21 \%$ and for $-8 \mathrm{kV}$ it drops from $90 \%$ to $69 \%$. This indicates that the surface potential decay depends on the ground electrode injection, which is field dependent.

\section{B. Corona Charging Current}

The corona charging current during the charge processes calculated by taking the measurement of the voltage across the resistor is shown in figure 3 . The current under $-3 \mathrm{kV}$ is too noisy to be read. The initial peak current is not shown in Figure 3 mainly because of very long charging time, however, our interest lies in the steady state of this current. This graph illustrates how the gold electrode affects the charge processes. Due to the bipolar behaviour of the charging progress, the measured current is the injection of holes from the bottom surface of the sample. This proves the field dependent injection from the bottom layer. 


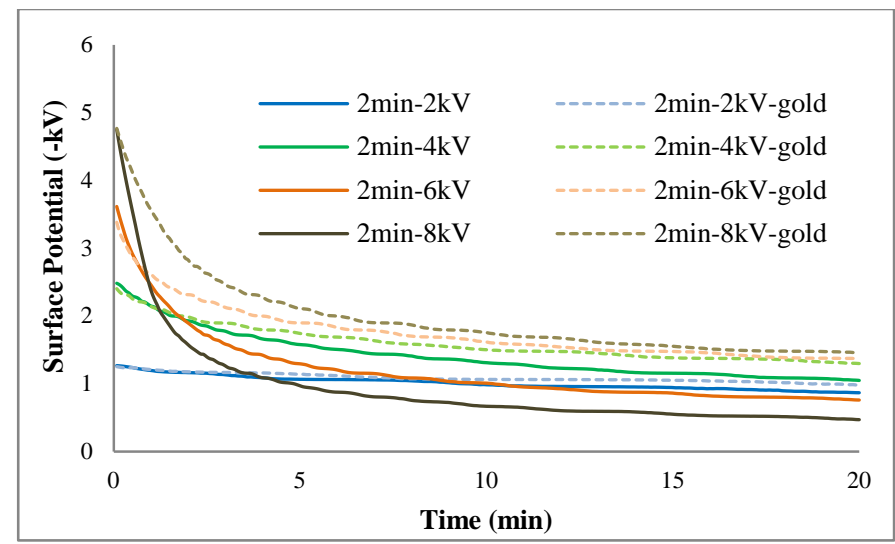

Figure 2. Surface potential decay for gold ground and normal ground LDPE film.

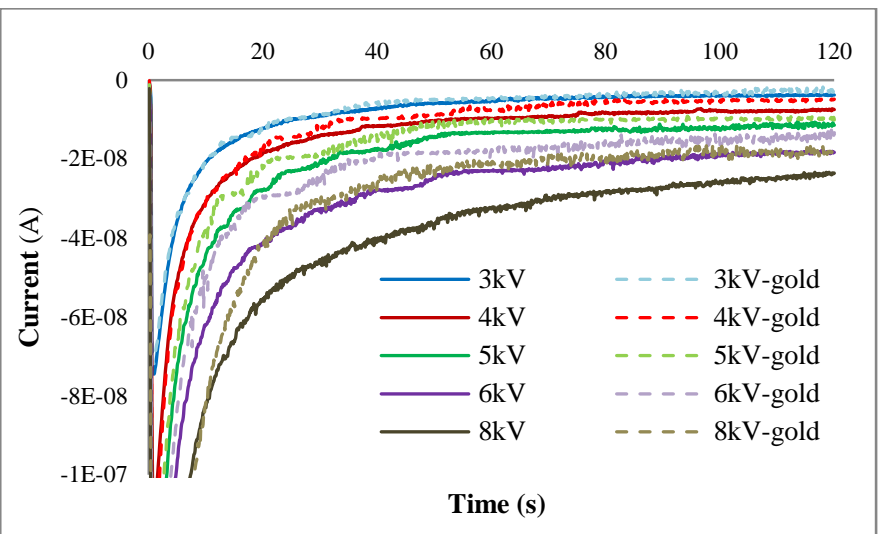

Figure 3. Corona charging current for gold ground and normal ground LDPE film.
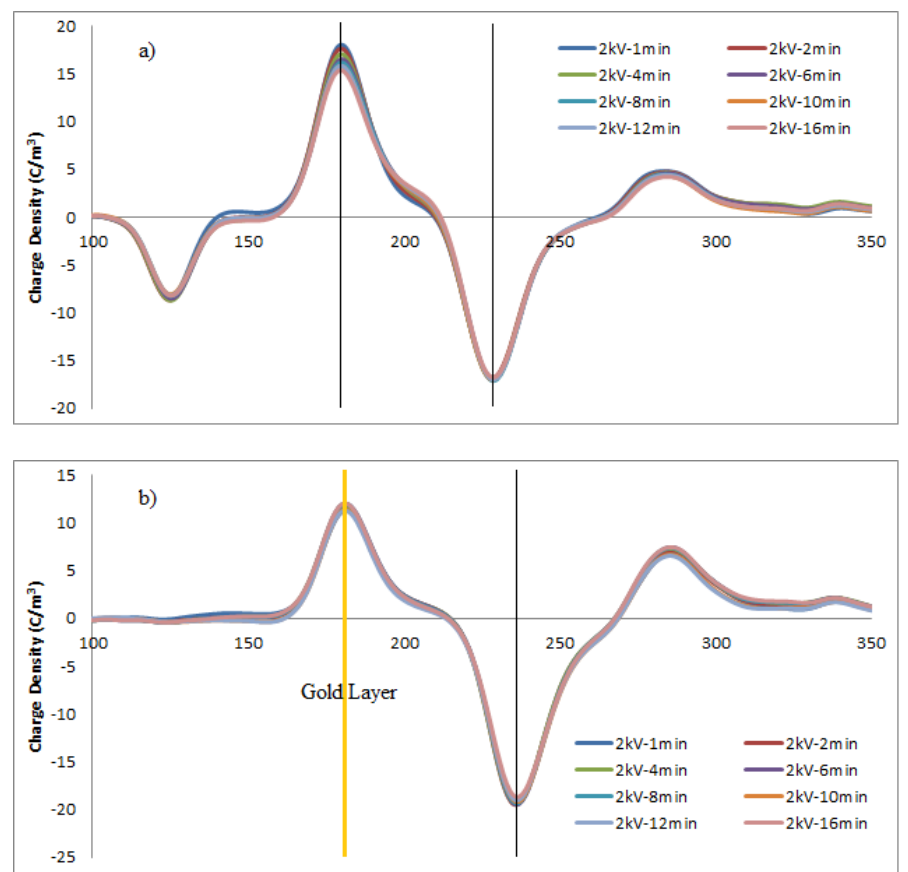
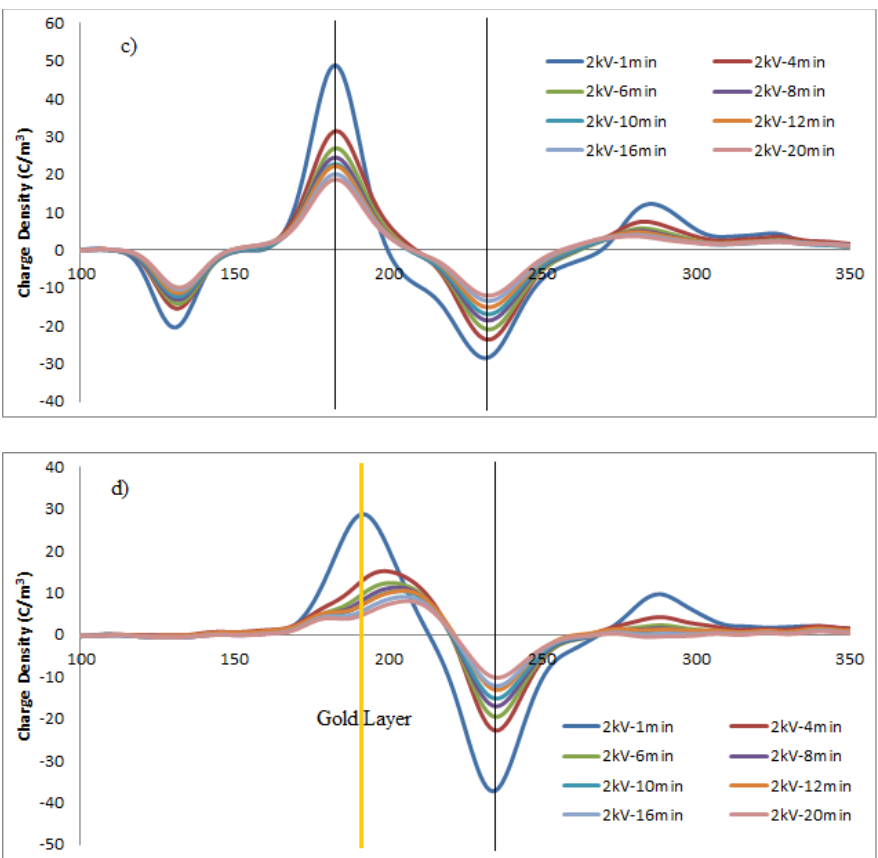

Figure 4. PEA results for a) $-2 \mathrm{kV}$, b) $-2 \mathrm{kV}$ gold ground, c) $-8 \mathrm{kV}$ and d) $-8 \mathrm{kV}$ gold ground.

\section{PEA Results}

The decay process observed by PEA is shown in figure 4 . The corona charged sample is sandwiched by 2 clean $50 \mu \mathrm{m}$ LDPE samples; it takes 1 min to transfer the charged sample into PEA and take the first reading. From figure 4, it can be seen that there is a big difference in the peak of positive charges on the bottom surface of corona charge sample (right vertical line) at different ground; there is also a difference in the negative peak (left vertical line) as well. It can be explained as the injection of holes is reduced, hence the number of positive and negative charges recombination is lower. This results more negative charges on the top surface after 1 min decay. The faster positive charge decay in the normal aluminum ground can be viewed from both $-2 \mathrm{kV}$ and $-8 \mathrm{kV}$ results, which prove the results in figures 2 and 3 .

\section{CONCLUSION}

In conclusion, it can be said that the experimental results from gold ground and normal ground LDPE films prove that the bipolar charge injection is the main mechanism for the surface potential decay on corona charged material. The charge injection from the bottom layer can play an important role in the surface potential decay process. A numerical model can be built based on our test data.

\section{REFERENCES}

[1] M. Ieda, G. Sawa and I. Shinohara, A decay process of surface electric charges across polyethylene film, J. Appl. Phys. $6793-4$

[2] Z. Xu, L. Zhang and G. Chen, Decay of electric charge on corona charged polyethylene, J. Phys. D: Appl. Phys. 40 7085-9 